\title{
INDAGACIÓN GENERAL SOBRE EL CONSUMO Y SUS EFECTOS EN LA SOCIEDAD'
}

(Recibido: Mayo 10 de 2012 Aprobado: Junio 13 de 2012)

Kabir Javier Corzo Rincón ${ }^{2}$ Luis Carlos Lezama Barreto ${ }^{3}$

\section{Resumen}

EI Análisis integral del fenómeno del consumo depara un apartamiento de los aspectos medibles del mismo, puesto que renunciar al estudio de este en términos estrictamente económicos es considerarlo como un fenómeno social e irreductible. De esta manera trascender y superar la visión de las mercancías de los economistas clásicos para comprender a estas como elementos que responden a los imaginarios sociales de una sociedad, es entender su valor signo y su renovación constante en los significado dentro de la modernidad. Es de esta manera, que podemos entender el papel de la publicidad y la mercadotecnia, pilares de la moda y de la obsolescencia moral de las mercancías las cuales establecen los patrones que enmarcan lo que se conoce como el consumismo: fenómeno que es inherente a la sociedad de consumo. La misma que ha permitido la aparición de la infraclase y un estado reflexivo a favor de una sociedad de consumo "ecológica". Sin embargo: ¿Es este el fundamento de la superación de la crisis planetaria?

\section{Palabras clave}

Sociología económica, imaginarios sociales, consumo, consumismo

\section{GENERAL INQUIRY ON GONSUMPTION AND ITS EFFECTS ON SOCIETY}

\section{Ahstract}

The Integral analysis of the consumption phenomenon implies a removal of its measurable issues, because refusing to study it in strictly economic terms is to see it as a social and irreducible phenomenon. In this way, transcending and overcoming the merchandises view of the classical economists, in order to understand these as elements that respond to the social imaginary of a society, is to understand their value as a sign and their constant renewal in the meanings within modernity. It is in this way that we can understand the role of advertising and marketing, fashion pillars and moral obsolescence of commodities which establish patterns that frame what is known as consumerism: a phenomenon that is inherent to the consumer society. This has also allowed the appearance of the underclass and a reflective state in favor of a "green" consumer society. However, is this the basis for the overcoming of the global crisis?

\section{Key words}

Economic sociology, Imaginary social, Consumption, Consumerism.

1 Artículo de investigación fundamentado en el estudio que realizaron los autores para acceder, bajo la forma de monografía, al título de economistas de la Universidad Industrial de Santander (UIS).

2 Economista de la Universidad Industrial de Santander. Ex integrante del cineclub microcine inútil. kabir-017@hotmail.com

3 Economista de la Universidad Industrial de Santander, Ex integrante de los cineclubes Microcine inútil y Cinerrante. Director de la 4ta edición de la revista Tlön de los estudiantes de la escuela de Economía. sankarlos@hotmail.com 


\section{Metodología y objetivos}

Para el siguiente artículo de investigación se realizó una revisión de contenido en términos sociológicos, tomados en cuenta los conceptos de imaginarios sociales y significaciones sociales, ya que desde este enfoque se puede hacer consideraciones más precisas frente a la categoría de consumo como la necesidad de alimentar un placer de representación. De esta manera se aparta de la visión establecida en el campo económico, del cual se han derivado sus estudios a lo estrictamente definido como las decisiones del consumidor, y se hace, en la medida que se considera que ésta realiza una simplificación absoluta, y una abstracción de la realidad de una categoría que tiene que ser estudiada como una variable social. Del mismo modo, parece apropiado evidenciar que hay elementos de la misma teoría económica, que deben ser trascendidos para un estudio sociológico, y de esta manera considerar, que la concepción de la teoría valor debe ser comprendida, también, como una teoría del valor signo. Desde esta concepción del estudio de consumo se puede entender las dinámicas que realizan tanto la publicidad y el marketing dentro de la configuración de los conceptos de imaginarios sociales y significaciones sociales, los cuales, a la postre, son el objetivo fundamental de una indagación general del consumo.

\section{Introducción}

Los aportes y las reflexiones que se presentan a continuación hacen parte del desarrollo posterior a la investigación que lleva por nombre: la sociedad de consumo contemporánea: "la búsqueda de la felicidad material. Donde, desde la sociología, se examina al consumo, sus imaginarios sociales y las instituciones, que bajo ciertas significaciones sociales, como el prestigio, el status, el confort, se materializan los sentimientos, bajo la satisfacción de un estricto placer de representación. De esta concepción del consumo devie- ne el análisis interdisciplinario propicio para entender en términos generales las concepciones tanto de consumo como de consumismo. Desde este punto de vista lo que podrá encontrar el lector a continuación, es una indagación general sobre aspectos claves para comprender en términos sociales el consumo.

\section{Aspectos iniciales}

Hablar del consumo como una mera variable cuantitativa sería sesgar una interpretación de éste a términos puramente nominales y una comprensión inocente cuando se obvian los efectos sociales que acarrea el acto de hacerlo no sólo a nivel personal, sino a nivel global. Decir que éste crece o disminuye no tendría sentido, más que en los economistas positivos, los cuales lo ven como una relación que se modifica de acuerdo a otras variables como el ingreso, el ahorro y la tasa de interés, como bien lo haría en su época (y aún en estos tiempos donde se ha resucitado en países en crisis) John Maynard Keynes.

A pesar que, en su lucidez, el señor Keynes expusiera en su libro de la teoría de la ocupación, todo un tratado para mantener la demanda efectiva, pilar de la ocupación, fundada en el corazón del consumo, por muchos años, siendo el enfoque de grandes potencias para desarrollar sus economías y salir de la trampa del desempleo. Hasta este punto el consumo funcionaba como un gran "engranaje dorado" que lograba articular y dinamizaba todos los aspectos de la vida humana. Sin embargo es evidente que los daños colaterales tanto ambientales como sociales son indudables, después de un siglo cargado de aceleración de la industria, obsolescencia programada de las mercancías y una cultura consumista que establece, mediante la moda, patrones que son rápidamente modificables. Pero para poder iniciar con este desarrollo posterior al análisis del mismo Keynes, debemos superar la definición de mercancía del mismo autor, puesto que hay elementos de 
juicio que no analizo, como el papel de las mercancías como objetos investidos de una lógica simbólica y de estos en función política de la ideología que con ella se establece (Baudrillard, 1972, 2), una idea más allá de la denominación realizada por los clásicos de la economía como meros valores en uso y en cambio. Este último aspecto se ampliará, al establecer tanto el papel de las mercancías como objetos revestidos de valor-signo; así como también el manejo de los medios de comunicación como reproductores mediante la publicidad y el marketing, para refinar, acentuar y renovar el lenguaje cultural dentro de la sociedad de consumo para hacerlo propicio para este. Con las ideas anteriores, se podrá entender cómo este lenguaje establece una renovación constante de las expresiones y sentimientos (especialmente la felicidad como significación material de la sociedad) que instan a los sujetos a agotar sus fuentes de ingresos en la materialización de ellos en lo que se ha considerado en la literatura como la democracia material, una institución formal que define la relación sujeto-mundo de una forma particular. $Y$ en este sentido, como el desarrollo mismo de la sociedad de consumo ha permitido la generación de la cultura consumista. De esta manera, es importante entender que las instituciones sirven de base para este cambio social que tenemos sobre el consumo ambiental, en toda una avanzada global, al establecer un proceso reflexivo que busca una transformación en el consumo como una significación social, que vaya de la mano con el cuidado del planeta y una garantía de suficiencia en términos de mínimos vitales para las personas. Sin embargo, en las últimas anotaciones se dejará a modo de cuestionamiento hacia donde debería orientarse tal cambio.

\section{Superar la visión del valor uso en las mercancías}

Hablar de los objetos metamorfoseados en mercancías como una demostración única y exclusiva del trabajo sin determinar lo que este significa frente a la relación sujeto-mundo, seria obviar que detrás de cada homo faber, se esconde un homo otiosus dispuesto a que su ocio le lleve a consumir. Sin embargo, no podemos obviar tampoco, que ese consumo se deriva de una coacción cultural que es determinada por un significado social del objeto. Es decir, cada objeto tiene un sentido social que sirve para discriminarlo, pues actúa como un diferenciador del sujeto frente a una sociedad claramente estratificada. Desde la época preindustrial esto ha servido para determinar el modo en el que se debe consumir el ocio en términos de ostentación. Esto lo conocemos desde Veblen en su teoría de la clase ociosa, cuando las clases aristocráticas definían un modo de vida destinado al ocio y a partir de él, orientaban más que un tipo distintivo de consumo, toda una moral del mismo en las clases dominantes: qué se debía, cómo se debía, y cuándo se debía consumir: todo una secularización del consumo. Secularización que no sólo determinaba una clase especifica, pues esta era irradiada en forma de simulación en las clases bajas.

En ese sentido, desde autores como Cornelius Castoriadis, podemos deducir que el mismo acto del consumo tiene dos fundamentos: el primero, satisfacer un placer de órgano (es decir, todo lo que necesita nuestro cuerpo para la subsistencia) y finalmente, un placer de representación, el cual tiene relación con la imaginación y lo que necesita nuestra psiquis para estar satisfecha (Castoriadis, 2004). En ésta se encuentra la coacción cultural y el consumo mediante significaciones sociales de acuerdo a un imaginario social impuesto mediante el establecimiento de la sociedad de consumo. Esto es importante, pues es allí en la psiquis, donde el individuo es más propenso a establecer su relación con el mundo, pues es donde la sociedad ejerce su poder y le hace una doma o lo condiciona actuar. De esta manera, imaginar que ya un objeto no 
representa en términos objetivos, única y exclusivamente, una función determinada a la hora de compra, sino que posee en sí, una diferenciación entre los iguales, empieza a establecer una lógica simbólica, la cual transmite una distinción, que no puede ser más que una forma de lenguaje y de un tipo particular de comunicación. De esta manera:

Cuando entablamos un diálogo social teniendo como intermediario la diferenciación y la estratificación, es decir, una jerarquización de las escalas de la sociedad, tomamos como referente al objeto que media entre los sujetos utilizando como lenguaje la significación social por el cual este objeto es revestido. Un ejemplo de ello son los automóviles de gama alta los cuales sirven como un elemento de diálogo social entre los sujetos de determinada escala social. Ese automóvil cuyo valor en uso es el de transportarnos a determinado lugar, cobra una noción diferente para el que fue creado, puesto que al diferenciarse por su marca de gama alta, ya no solamente posee un valor en uso, sino que ahora es revestido con un valor signo (Corzo; Lezama, 2012).

El anterior, es un ejemplo trivial si hablamos en términos específicos lo que representa este objeto-signo, metamorfoseado en mercancía y asumido como un elemento de lenguaje dentro de la relación de los sujetos en la sociedad de consumo. Nada diferente a lo que vivimos en nuestra cotidianidad, donde se materializan las emociones y los sentimientos hasta traspasar la barrera de lo subjetivo e instaurarse en toda una medición objetiva de la vida humana: "dime cuanto consumes y te diré que tan feliz eres", diría el dicho popular. Y aunque no está demás, hacer mención de esta cualidad del objeto-signo debemos hacer precisión que esta hace parte de una lógica que sólo tiene sentido, cuando el objeto como tal, autónomo en cuanto a su signo, logra ser diferenciado por otros sig- nos. Extraña apreciación de la mercancía que es pasada por alto, ya que no todos los objetos nos pueden representar prestigio, o felicidad, sería una visión totalizante del consumo. De ahí que devenga el papel del consumo también como contracultura, pero al mismo tiempo parte de la misma sociedad de consumo, en pocas palabras un juego cimentado entre los antagonismos pero con un mismo fin.

Ahora bien, si entablamos el debate de estas mercancías (objetos-signos), en términos de control de una sociedad, instauraremos un debate parecido en esencia a lo que encarnan las ideas Keynesianas, puesto que no puede obviarse de forma singular las representaciones simbólicas de las mercancías, sin hablar de estas también como una función política de la idea capitalista de acumular riqueza y crear las nociones culturales del sujeto de consumo y entablar, en términos más extensos, lo que es una lógica de consumo. Sin embargo, nos remitiremos a continuación en términos generales a lo que significa el consumo y el consumismo.

\section{Indagación general del con- sumo}

El consumo, en su más simple expresión, ha estado directamente ligado a la actividad humana. El consumo en sí mismo es necesario para la vida, el hombre debe comer para mantener su cuerpo en funcionamiento, debe disponer de refugio y vestido para afrontar el clima, en ese sentido el ser humano debe disponer de una serie de bienes y recursos para afrontar unas necesidades básicas individuales. ¿En qué momento el consumo pasó a transformarse en un problema, más conocido actualmente como consumismo? Para Bauman, el consumo y el consumismo depende del contexto donde cada uno se desenvuelva, en ese sentido expone: "a diferencia del consumo, que es fundamentalmente un rasgo y una ocupación del individuo hu- 
mano, el consumismo es un atributo de la sociedad" (Bauman, 2007, 47).

Se sostiene entonces, que el consumo ligado a los individuos no representa problema, éste surge cuando las necesidades básicas de los individuos son adoptadas por la sociedad, pero la sociedad no puede responder de manera individual a cada uno de sus integrantes, por esta razón tiene que estandarizar las necesidades y crear parámetros de satisfacción, en ese orden de ideas, al generalizar parámetros de consumo se aliena al individuo de sus necesidades y estas pasan a pertenecer a la sociedad, en el momento en el que la sociedad toma las necesidades de los individuos para ella misma aparece el consumismo.

Para iniciar, se debe mencionar tres aspectos especiales para el establecimiento del consumismo y, por ende, la creación como tal del síndrome consumista: lo primero que se debe mencionar es la idea del sujeto competidor, que es inherente a la premisa de ser siempre los primeros, es decir: "estar y mantenerse a la delantera" en el pelotón de lo novedoso, de las tendencias $\mathrm{y}$, por ende, mantener una lenguaje actualizado en el consumo de mercancías. Esto con el fin de estar en el grupo de referencia y asumir un rol de autorreferencia ${ }^{4}$ en la sociedad de consumo. Desde este punto de vista, estamos de acuerdo con las palabras de Michel Maffesoli cuando dice: "soy quien soy porque los otros me reconocen como tal" (Bauman, 2007, 115). Que traducido a nuestro lenguaje sería como: "Soy lo que compro porque en mi grupo todos me reconocen como importante". Y en este sentido, es donde la relación sujeto-mundo frente al "pelotón de la moda" cobra valor en la medida que guarda una relación estrecha con las marcas, el significado de las mismas (es decir, toda la concepción del

4 Ésta consiste en poder diferenciar una cosa de otra dada, en una serie de parámetros establecidos, podría decirse que un sujeto tiene auto-reflexividad al distinguir entre el bien y el mal dada una construcción moral anterior. objeto signo) y el seguimiento constante de ellas. El sujeto auto-referenciado ahora utilizará al mercado para que medie con sus lazos sentimentales y la concepción de fraternidad quede enlazada en un círculo netamente material. Si el sujeto hiciera todo lo contrario y decidiera no adquirir las mercancías que contienen un alto valor simbólico, estaría frente a la palestra pública de la sociedad, pues esta actuaría como un elemento de rechazo que produce un apartamiento del sujeto, por ello, la mejor elección la hará el sujeto al comprar los objetos necesarios para poder alcanzar su felicidad material. Es este sentido de "pertenencia" social en la que se anida, como tal, la cultura consumista. Sin embargo, en esta carrera por aprobación e inclusión el sujeto sabe que todo lo que compre tiene una clausura social, es decir, una obsolescencia moral. Este es quizás el segundo elemento clave para entender una cultura consumista, puesto que ya no basta con estar en la competencia por la delantera de lo novedoso sino mantener como tal, la promesa viva de un cambio constante de la alegría material. Esta capacidad de respuesta temprana por parte del sujeto, no solo le da una ventaja frente a su grupo, sino también una sintonía con la velocidad del conjunto de instituciones que de antemano le dicen: "no hay que perder el tiempo". No obstante, el sujeto sabe que esta promesa cautiva (si así se le puede llamar) de mantenerse a la delantera de la innovación trae consigo una trampa y es, precisamente, la de su corto tiempo de satisfacción. Esto último, habla también de la euforia que trae consigo el sujeto consumista, como si se tratara de una droga que necesitara estar constantemente en consumo para mantenerse con la promesa de una realidad material donde lo único que importa es ser aceptado. De esta manera, es justificable para ello, seguir con el juego entre obsolescencia-innovación o de velocidad y olvido. Sin embargo, no sólo estas dos ideas, la de aceptación social y de renovación permanente son las promotoras del consumismo, también existe 
una tercera idea y es, precisamente, la de una "falsa libertad" al momento de elegir las mercancías, puesto que se le muestra al sujeto una alta variedad de estilos, este queda encerrado en uno solo y con él su rango de consumo. Mirándolo desde una esfera inferior, al pertenecer a un grupo, esto ya tiene inmerso una estructura, en el sentido de mantener un prototipo de sujeto a modo de escaparate capaz de establecer lo que debe ser consumido y, a su vez, servir de referente, en la carrera por la inclusión. De esta manera como diría Bauman: "elegir, en sí (...) no es el tema, pues es lo que uno debe hacer bajo pena de exclusión" (Bauman, 2007, 118). Es decir, el sujeto no tiene la libertad de modificar los patrones, sólo reproducirlos y, si no lo hace, está destinado a ser desterrado del grupo. De esta manera, el sujeto no ve esto, pues en la medida que usa su tiempo para seguir a la delantera de la innovación, manteniendo una idea de éxito como significación social y una libertad coartada por la idea de no exclusión, se enjaula en un programa de vida totalmente coherente con las metas propuestas por la sociedad de consumo, es decir, toda una vida para consumir de manera inmutable; para no desfallecer en la materialidad de sus emociones. Pero, a pesar de ser un estilo de vida, también es un síndrome puesto que, y según Bauman:

el síndrome consumista ha degradado la duración y jerarquizado la transitoriedad y ha elevado lo novedoso por encima de lo perdurable. Ha reducido abruptamente el lapso que separara no solo las ganas de su satisfacción (...), sino también el lapso entre el momento del nacimiento de un deseo y el momento de desaparición, así como entre la conciencia de la utilidad y el beneficio de las posesiones y la sensación de que son inservibles y dignas de rechazo (Bauman, 2007, 120).

Es así, que al momento de señalar a la sociedad como responsable tanto del consumismo como de su síndrome, se debe hacer un análisis de la misma y debe preguntarse, entonces: ¿Qué características de la sociedad son factores esenciales para el consumismo? La sociedad, como se conoce hoy en día, debe gran parte de sí misma a la revolución francesa, es así como los principios de igualdad, libertad y democracia que parten de dicha revolución se manifiestan en la sociedad contemporánea a favor del consumismo.

Para entender el papel de los conceptos anteriormente mencionados en la consolidación del consumismo debemos regresar a las necesidades básicas del individuo y buscar aquella capaz de hacer todo posible en tal sentido.

La felicidad del individuo, considerada como la búsqueda de un estado deseado, puede ser vista como el motor de las acciones de cada individuo para lograr lo que se desea, independientemente de lo que sea. La sociedad, para estandarizar la necesidad de felicidad (diferente en cada uno de los individuos) recurre a significaciones y signos que la representen, sea acumulación de riqueza, monumentos, dioses, vida después de la muerte y finalmente: objetos. En ese orden de ideas, necesita una unidad de medida que le respalde y aquella que no puede ser respaldada simplemente se rechaza "Mientras tanto, la felicidad como goce total o interior, esa felicidad independiente de los signos que podrían manifestarla a los ojos de los demás, esa felicidad que no tiene necesidad de pruebas, queda pues excluida de entrada" (Baudrillard, 2009, 39)

La felicidad medida en objetos es la adoptada por la sociedad para suplir la que los individuos buscan. La felicidad material inscrita en una sociedad libre, igualitaria y democrática se manifiesta de la siguiente manera: frente a los ojos de la sociedad todos son iguales, así mismo son iguales frente a los objetos que otorgan felicidad, pero los objetos gozan de dos características en el sentido Smithiano -valor en uso y 
valor en cambio- frente a la primera los individuos son iguales, el vestido, la comida, el automóvil cumplen necesidades específicas; protección contra el clima, alimento y transporte respectivamente, pero es en la segunda característica donde la igualdad no se cumple. El valor en cambio que está regido por las leyes de oferta y demanda, excluye a aquellos que no tienen el ingreso suficiente para adquirir el objeto deseado que le proporciona la felicidad material anhelada dentro de la sociedad.

Ahora bien, la sociedad en su conjunto de reglas es una sociedad democrática e igualitaria, todos los individuos tienen las mismas posibilidades de alcanzar la felicidad material y son iguales ante el estado en la medida de sus capacidades, en teoría, todos tienen acceso al auto de lujo, el problema radica en los ingresos de cada individuo para obtenerlo y esa diferenciación de ingresos no es problema de la sociedad, dado que eso depende de qué hagan los individuos en el ejercicio de sus libertades y capacidades.

Si bien la sociedad enmarcada en la búsqueda de la felicidad material se basa en principios de igualdad y democracia, estas características sólo aplican a una sola faceta de los objetos: el valor en uso. Frente al valor, en cambio, todo está en manos de las leyes del mercado y, por ende, allí no aplican tales conceptos de igualdad o democracia.

El fallo institucional frente al valor en cambio de los objetos genera lo que Baudrillard concibe como "diferenciación de clase" $(2009,57)$ la cual consiste en la desigualdad llevada por fuera de las fronteras del consumo de objetos, esta desigualdad también se presenta en la educación, la cultura y así mismo las oportunidades. La desigualdad trasciende a los objetos al igual que el consumismo, Bauman (2007, 162) señala: "la transformación total y absoluta de la vida humana en un bien de cambio". Es decir, todo lo que se contempla en la sociedad está sujeto, por ende, a la desigualdad o restricción a su acceso. En ese orden de ideas aparece un peldaño en descenso dentro de las clases anexas a la diferenciación social, aparece lo que Bauman llama "la infra-clase".

La infra-clase está compuesta por aqueIlos individuos que no pueden vivir el consumismo de la misma manera que el resto de la sociedad, dada la desigualdad de ingresos y fallos en la toma de decisiones que los dejan por fuera de la búsqueda de la felicidad material, en esta esfera encontramos, a los desempleados, inmigrantes ilegales, indigentes, madres solteras y todas esas personas a las cuales el Estado debe poner especial atención dado que no pueden valerse por sí mismas. Estos "consumidores fallidos" (Bauman, 2007, 168) son señalados como sinónimos de maldad y perversión dado que se alejaron del consumismo, es decir, de la búsqueda de felicidad material a la que toda la sociedad está ligada, por ende, deben ser vistos con malos ojos y temérseles.

A pesar de ser víctimas de un estado de desigualdad, la sociedad ve a la infraclase como individuos que están ahí por elección propia, dado que si hacen un mayor esfuerzo, estos individuos pueden volver al consumismo (al menos en niveles bajos) y considerarse así como parte de la sociedad. Bajo este argumento, la pobreza es un estado normal de la sociedad y el Estado no tiene que hacer mayor esfuerzo para solucionarla, dado que eso depende de cada uno de los individuos.

\section{La publicidad en el consumo}

La sociedad contemporánea ha sido testigo de la aparición de las tecnologías de la información, de la transmisión de datos en tiempo real y la masificación de los medios de comunicación. Este conjunto de avances técnicos y tecnológicos, permite a las personas mantenerse conectadas con el resto del mundo independientemente del lugar en el que se encuentren, de esa manera, lo que 
ocurre en el otro hemisferio del planeta puede repercutir directamente en nuestro hemisferio. Las empresas y firmas dedicadas a la producción de bienes y servicios se han visto beneficiadas por aquellos avances y de la mano de la publicidad han llevado el mensaje de la felicidad material a todos los rincones del globo. Pero la publicidad como la conocemos, hoy en día, no aparece de manera espontánea en el mundo, esta nace a partir de una construcción histórica ligada a las formas de producción.

Con la aparición del Taylorismo y el Fordismo, la producción en masa de bienes de consumo se hace patente en el mundo, en virtud de la aceleración de la velocidad de producción los bienes fabricados no gozaban de rasgos diferenciados entre ellos, por tanto, el papel de la publicidad en aras de la renovación constante de inventarios se dedicaba a la promoción excesiva del producto mediantes avisos y comerciales que trascendían los límites de tolerancia y se tornaban invasivos para el consumidor, creando en ellos la necesidad de obtener el bien (Lipovetsky, 2007, 61).

Dado que los bienes producidos a principios del S. XX gozaban de un ciclo de vida relativamente largo, las empresas comienzan a implementar métodos de obsolescencia por medio de la baja de calidad en los bienes y cambios en la manera de hacer publicidad. En ese sentido, la publicidad deja de abalanzarse sobre los consumidores y, por el contrario, empieza a entablar diálogo con ellos en el orden de permitir a los consumidores el acceso a bienes diferenciados.

La publicidad empieza a apelar entonces a lo que Castoriadis Ilama "placer de representación" (Castoriadis, 2004, 29), es decir, esa energía psíquica que sobreviene del placer de órgano. El diálogo que la publicidad entabla con el individuo desencadena lo que hoy conocemos como marketing, ese "arte" de descubrir qué mueve a cada individuo a comprar un bien, mediante el marketing se permite entonces buscar qué necesidades de representación tiene un individuo determinado para luego, por medio de la publicidad generar ese valor signo en el objeto que hace que sea más apetecido por el individuo. Hoy en día, las marcas y eslóganes hacen que el individuo se relacione directamente con una firma determinada, haciéndose fiel a una marca o una determinada gama de productos.

El último estadio de la publicidad pone a la esfera de la producción al servicio del consumismo, mediante un diálogo más íntimo con el individuo en una sociedad dominada por los Mass Media los objetos que proporcionan felicidad son, cada vez, más propios e individuales, la personalización del producto desde el momento mismo de su producción permite a los individuos poner todos los signos necesarios al objeto deseado, sean estos signos: prestigio, confort, estatus y demás. Esta ilusión de libertad sobre los objetos deseados es la mejor forma de atracción que puede brindar la publicidad, dado que el individuo a final de cuentas está sujeto a la gama de opciones que la publicidad brinda y estas en algún momento llegan a un límite, Lipovetsky expresa esta premisa en el sentido de que "la publicidad propone, el consumidor dispone" (2007, 169).

En ese orden de ideas, la masificación de los medios de comunicación ha permitido llevar el consumismo a todos los rincones del mundo y generado en las personas la necesidad de comprar para ser felices. Es en este momento cuando se pone de manifiesto el poder de los medios de comunicación para influir en el individuo y, a su vez, surge la duda de si podría darse vuelve atrás en la carrera consumista y llevar a la sociedad a un modelo de consumismo menor o nulo. 
Assadourian (2010) parte del hecho que el consumismo ya está arraigado en la cultura, es decir: para las personas que nacen hoy en día el consumismo es algo normal, sin siquiera tener uso de razón el individuo está sometido al bombardeo constante de pautas publicitarias y avisos comerciales que le brindan una imagen de lo que se concibe como mundo -pueden encontrarse estudios de niños que a temprana edad reconocen más logotipos y marcas que el nombre de las empresas-. El autor enumera entonces una serie de instituciones responsables de la normalización del consumo y su arraigo en la cultura.

En primer lugar, se presenta al mercado como principal instrumento de culturización consumista y la publicidad como gran aliado y herramienta, dado que por medio de la publicidad el mercado controla los deseos de los individuos frente a los objetos que consumen. En segundo lugar, se encuentran los medios de comunicación como principales vehículos de todas las pautas sociales, signos, representaciones y aspiraciones necesarias y básicas para que el consumismo se consolide en la cultura.

En tercer lugar, se responsabiliza al gobierno en su papel de generador de normas en instituciones rígidas, dado que éste se ha subordinado al mercado al permitir las características anteriormente mencionadas, como manipulador de signos y demás, por otra parte, el gobierno en su afán de liberar el mercado y buscar la creación de Estados neoliberales "eficientes" se ha sometido al yugo de las empresas, hasta llegar a extremos como el del discurso de George W. Bush en el marco de los atentados del 9/11 donde instó a sus compatriotas a superar el duelo y la pérdida por medio de la compra de bienes.

En cuarto lugar, pero no menos importante, se encuentra la educación, no tanto bajo el carácter de aliado, sino más bien, en el papel de institución permisiva y acrítica. La educación, por su parte, debería por lo menos emprender el debate de si el consumismo es o no el modo de vida que mejor se adecúa a la sociedad. Pero en su lugar la educación ha sido permeada por el mercado "desde materiales educativos hasta patrocinio de disciplinas del conocimiento" (Assadourian, 2010, 52).

Las instituciones anteriormente mencionadas se han encargado de normalizar el consumismo en la cultura y son ellas las que tienen la posibilidad de cambiar el rumbo de la misma. Sin embargo, Se debe llegar a un proceso "reflexivo" para de esta manera sacar al mundo de la idea de consumo desenfrenado en el que se ha visto envuelto.

\section{Epílogo del consumo}

Hasta este punto se ha tratado el tema en términos generales con un acercamiento en términos sociales del mismo. Pero establecer ese proceso "reflexivo" trae consigo, el establecimiento de que la realidad tal y como la conocemos, por lo menos en este plano, en términos de biosfera no va por buen camino. Hablar de un proceso "reflexivo" en términos de Castoriadis significa una crisis del ente suprasensible; la idea o razón dominante, entendida como la que orienta y explica bajo una lógica la relación del sujeto y el mundo en el tiempo. Si la razón de nuestra sociedad capitalista es la acumulación, mediado por el mercado, y el desarrollo de las relaciones de producción en pos de un progreso ilimitado. Entender que el establecimiento de la sociedad de consumo es una manifestación de ello, es una evidencia de la esencia del problema, es decir, la producción incesante de objetos en favor de un crecimiento material ilimitado. De lo anterior deviene, todo el desarrollo que se realizo en términos culturales en el siglo $X X$. Y desde este punto de vista, preguntarse por la esencia del 
problema y no por las manifestaciones del mismo es importante, puesto que la sociedad contemporánea solo enuncia cambios frente a las manifestaciones. Es decir, ahora tendremos que consumir en términos de cuidado de la naturaleza, con empaques "ecológicos", y filosofía de vida medida en logos que "cuidan el planeta", mientras se mantiene una industria que aumenta constantemente la extracción de materias primas de la tierra, para abastecer y satisfacer los cada vez mas placeres de representación que se originan y se renuevan en el consumo. ¿Pero acaso el hombre es el ultimo bastión frente al cuidado del planeta?: no! Esa frase pareciera una estrategia más de mercadotecnia, que un mensaje alentador por cambiar la concepción de este sujeto de consumo frente al mundo. $Y$ esto es así, porque en el discurso mediático no está la interrogante sobre el consumir menos, sino consumir de otra manera. El rescate pues, esta sobre el sujeto que se continúa reproduciendo en su relación con el planeta, es decir, el rescate debe ser sobre la humanidad. En síntesis, una pregunta por la relación hombre-mundo por encima de una sociedad de consumo "biodegradable": ¿es la reproducción de este sujeto consumista una salida a la sustentabilidad del planeta?

\section{Conclusiones}

El consumo a nivel de sociedad no puede ser tratado solo como una variable mas dentro del aparato económico, necesita ser observado bajo una perspectiva multidisciplinar que pueda arrojar una visión mas amplia del consumo y por ende un tratamiento más acertado al mismo. Como fundamento inherente del individuo ha sido sustraído de la psique para incorporarse al imaginario social y de esta manera transformarse en consumismo, un individuo dentro de una sociedad no consume solamente en afán de satisfacer sus necesidades básicas, es decir "placer de cuerpo" (Castoriadis, 2002). Lo hace en mayor proporción para satisfacer el placer de representación y por ende aparece lo que Baudrillard llama: valor signo de los objetos.

El consumismo como lo conocemos en la actualidad es el resultado de un proceso histórico social, que se ha manifestado con mayor fuerza desde la aparición del sistema capitalista y el desarrollo de las fuerzas de producción tales como el Fordismo y Taylorismo. En ese orden de ideas el consumismo y los imaginarios sociales que le sustentan están ligados a sistemas económicos y productivos que han alienado del individuo de sus necesidades básicas y les han impuesto unas nuevas, tales como: el status, confort, prestigio y demás.

El mercado y las empresas, por medio de la publicidad, han logrado permear todas las esferas de la sociedad con una amalgama de necesidades de representación, y a su vez, dejando de lado aquellas consideradas básicas. Por su parte los gobiernos, medios de comunicación y sistemas educativos han contribuido por acción u omisión al fomento del consumismo como modo de vida de la sociedad.

\section{Referencias}

Baudrillar, J. Critica a la economía política del signo. Edit Siglo XXI editores. México, 1983

Baudrillard, J. La sociedad de consumo: sus mitos, sus estructuras. Trad A. Edit Siglo XXI. España 2009

Bauman, Z, vida de consumo; trad, de Mirta Rosemberg y Jaime Arrambide. Edit, Fondo de cultura economía, Mexico. 2007

Castoriadis, C. Sujeto y verdad en el mundo históricosocial. Fondo de cultura económica. Argentina 2004

Corzo,L. (2012).La sociedad de consumo contemporánea: la búsqueda de la felicidad material. Universidad Industrial de Santander, Bucaramanga-Colombia 2012

Lipovetsky, G. La felicidad paradójica: ensayo sobre la sociedad del hiperconsumo. Traducción: Antonio, Prometeo Moya. Editorial Anagrama. Barcelona, 2007. 